RESULTS: This phenomenon may involve epilepsy induced excitation of the thalamus. This then acts to reduce the fidelity of retinogeniculate transmission and increase "signal to noise ratio" in visual transmission. This may contribute to complex visual hallucinations with eyes open. The hallucinated figures becoming clearer with eyeglasses provides support that this complex hallucination arises in the pathway from retina-LGN-cortex, not from stored visual associated cortex of top-down cortical release.

CONCLUSIONS: Given the above, those with visual hallucinations should be queried as to the influence of refraction on the clarity of hallucination.

FUNDING: No funding.

120

\section{Fragile X Syndrome Sharing Similar Neural Network Abnormalities as ADHD}

Chunhui Yang, $M D, P h D^{\prime}$; Carolyn Beebe Smith, Ph.D. ${ }^{2}$, Guoqiang Xing, $M D, P h D^{3}$; and Sandeep Gaonkar, $M D^{4}$

${ }^{1}$ Conventions Psychiatry \& Counseling, Naperville, IL

${ }^{2}$ NIH Cerebral Metabolism Building 35 Room 2 B1016

35 Convent Drive MSC3702 Bethesda MD

${ }^{3}$ Department of Psychiatry, Uniformed Services

University of the Health Sciences, Bethesda, MD

${ }^{4}$ Conventions Psychiatry \& Counseling, Naperville IL

ABSTRACT: Title: Fragile $\mathrm{X}$ syndrome sharing similar neural network abnormalities as ADHD

STUDY OBJECTIVE(S): The Fragile $\mathrm{X}$ syndrome (FXS) phenotype typically involves a variety of psychiatric symptoms, including features of autism, attention deficit/hyperactivity disorder (ADHD), anxiety, and aggression. Studies have shown that ADHD is characterized by multiple functional and structural neural network abnormalities including fronto-striatal, fronto-parietotemporal, fronto-cerebellar and fronto-limbic networks (Rubia, 2014; Norman, 2017). Studies have shown that $\mathrm{ADHD}$ is characterized by a delay in structural brain maturation (Rubia, 2007). Absence of the FMR1 gene product Fragile X mental retardation protein (FMRP) results in FXS, an inherited form of mental retardation. FMRP is an RNA binding protein functioning as a nucleocytoplasmic shuttling. In a knockout mouse model of FXS (Fmr1 null), Qin, et al showed regionally selective effects on cerebral metabolic rates for glucose (rCMRglc) (Qin, 2002) and rates of cerebral protein synthesis (Qin, 2005). In the present study, we asked if there is a relationship between brain regions most vulnerable to the effects of the absence of FMRP in the Fmr1 null mouse, and if the distribution consistent with the structural and functional brain abnormalities in ADHD. We also asked if there is a difference between males and females in the regional distributions and the levels of the FXR mRNAs.

METHOD: We used 35S-labeled probes specific for the mRNAs to perform in situ hybridization on brains from male $(n=4)$ and female $(n=4)$ mice at 6 months of age. Flowing hybridization, brain sections were exposed to $\mathrm{X}$-ray film and optical density were measured in nine brain regions on autoradiograms of sections hybridized to the probe.

RESULTS: The highest levels of expression we observed were in the cerebellum, granular layers of the hippocampus. Levels of expression were also high in CA1 pyramidal neurons of hippocampus, amygdala and granule layer of olfactory bulb. We found intermediate levels in the anterior hypothalamus and in cingulate and frontal cortex. Low levels of expression were found in thalamus and caudate. The distribution for the probe was similar in male and female mice, but we found a tendency for male mice to have higher levels than females.

FUNDING ACKNOWLEDGEMENTS: No funding.

121

\section{Enhancing Emotional Wellness With Smartphone Apps in Early Psychosis}

Dale D'Mello, M.D.

Associate Professor Emeritus, Department of Psychiatry, Michigan State University, East Lansing, MI

ABSTRACT: Introduction: There is wide consensus that the combination of psychopharmacology and psychotherapy is superior to either approach used alone, in managing depressive, anxiety and psychotic disorders. Completing homework assignments are prerequisite for successful outcomes in psychotherapy. The convergence of digital computer technology and consumer selfempowerment have generated a bewildering array of mental health applications for smartphones and other mobile devices. The purpose of the present poster is to review available apps of interest for patients with early psychosis.

METHOD: A search was recently conducted of the stores on the iOS and Android platforms, seeking apps for mood, anxiety, psychotic and cognitive disorders. Reviews of digital technology resources provided by the International Mental Health Research Organization (IMHRO) at www.psyberguide.org were consulted. Criteria for inclusion included: (1) popularity measured by greater 
than 10,000 downloads (2) a score of 3.5 or higher on the Mobile Apps Rating System (MARS) and (3) acquisition cost less than $\$ 1$. Consumer reviews were scanned. A total of 7 apps were culled from an expanding universe of thousands. This included top-rated apps in each of three symptom domains: depression, anxiety and cognitive disorders. Ranked in order of MARS rating the leading depression apps were "Depression CBT Self-Help Guide" and "eCBT Mood". The most popular anxiety apps were "Stop Panic \& Anxiety" and "Headspace". The top apps for cognitive enhancement training were "Brain HQ" and "Fit Brains Focus". In addition, the suicide prevention app "My3" was included because of its life saving potential. Consumers have rated the reviewed apps favorably. Conclusion: Smartphone apps are achieving wide acceptance in self-management of common psychiatric disorders. Clinicians need to become familiar with these adjunctive therapeutic tools, and integrate them in brief psychopharmacology visits.

FUNDING ACKNOWLEDGEMENTS: No funding.

\section{2}

Use of Pimavanserin in Patients With Parkinson's Disease Psychosis: Subgroup Analysis of Efficacy and Safety in Patients With and Without Cognitive Impairment

Daniel Weintraub, $M D^{\prime}$; James Norton, PhD ${ }^{2}$; Bruce Coate, $\mathrm{MPH}^{3}$; Candace Andersson, $\mathrm{PhD}^{4}$; Doral Fredericks, $M B A$, PharmD ${ }^{5}$; and Clive Ballard, $M D^{6}$

${ }^{1}$ Professor of Psychiatry and Neurology, Perelman School of Medicine at the University of Pennsylvania, Philadelphia, PA

${ }^{2}$ Sr. Dir. Medical Affairs, ACADIA Pharmaceuticals Inc., San Diego, CA

${ }^{3}$ Associate Director Biostatistics, ACADIA

Pharmaceuticals Inc., San Diego, CA

${ }^{4}$ Sr. Medical Science Liaison, ACADIA Pharmaceuticals Inc., San Diego, CA

${ }^{5}$ Vice President Medical Affairs, ACADIA

Pharmaceuticals Inc., San Diego, CA

${ }^{6}$ Pro-Vice-Chancellor and Executive Dean, The University of Exeter Medical School, Exeter, UK

ABSTRACT: Objective: A planned subgroup analysis of a phase 3 study was performed to evaluate the efficacy and safety of pimavanserin (PIM) in Parkinson's disease psychosis (PDP) patients withglobal cognitive impairment.

BACKGROUND: PDP is frequent, distressing, a leading cause of institutionalization, complicates PD management and is linked to increased morbidity, incident dementia and mortality. PIM, a selective serotonin receptor (5-HT2A) inverse agonist/antagonist, is newly FDA-approved for the treatment of hallucinations and delusions associated with PDP.

METHODS: In Study 020, a 6-week FDA registration study, 199 patients with baseline Mini-Mental State Examination (MMSE) score $\geq 21$, moderate-severe psychosis, and on stable PD meds, were randomized to PIM (34 mg/day) or placebo (PBO) for 6 weeks. This subgroup analysis evaluates efficacy and safety between two groups: those with MMSE total score $\geq 21$ but $<25$ (cognitively impaired; equivalent to Montreal Cognitive Assessment [MoCA] score 15-19) and those with score $\geq 25$ (cognitively normal; equivalent to MoCA score 20-30). Safety assessments were performed on the full safety dataset (i.e., three 6-week placebo-controlled studies) including 614 subjects $(\mathrm{PIM}=382, \mathrm{PBO}=231)$.

RESULTS: Overall, patients in the PIM group experienced a statistically significant improvement in SAPS-PD scores from baseline to Day 43 compared with PBO $(-5.79$ vs. $-2.73 ; p=0.001)$. In the subgroup analysis stratifying by baseline MMSE score, the change from baseline to Day 43 compared with PBO in the cognitivelyimpaired group $(\mathrm{N}=50)$ was numerically larger $(-7.11 \mathrm{vs}$. -0.47; $p=0.002)$. In the full safety dataset examining cognitively impaired patients, there were no betweengroup (PIM vs. PBO) differences in any treatmentemergent adverse event (TEAE) $(57.6 \%$ vs. $56.1 \%)$ or serious TEAE $(6.8 \%$ vs. $5.3 \%)$. The most common TEAEs occurring at $\geq 5 \%$ in either group were fall (7.4\% vs.10.5\%), confusional state $(6.5 \%$ vs.1.8\%), and orthostatic hypotension $(0.0 \%$ vs. $8.8 \%)$.

CONCLUSIONS: In this subgroup analysis of PDP patients, the treatment effect of PIM on SAPS-PD was larger in the cognitively-impaired group, with similar TEAE and serious TEAE rates. These results hold promise for cognitively-impaired patients that will be further elucidated in future studies.

FUNDING ACKNOWLEDGEMENTS: Clinical study was funded by ACADIA Pharmaceuticals Inc.

\section{3}

\section{Adjunctive Brexpiprazole in Patients With MDD} and Symptoms of Anxiety: Results From Post-Hoc Analyses of Three Placebo-Controlled Studies

Emmanuelle Weiller, PsyD'; Anna-Greta Nylander, PhD ${ }^{2}$; Catherine Weiss, $P h D^{3}$; Peter Zhang, PhD ${ }^{4}$; and Mary Hobart, $M D^{5}$

${ }^{1}$ Lead Medical Advisor, Medical Affairs, H. Lundbeck A/S, Valby, Denmark 